\title{
Development of malignancies and their outcomes in patients supported on continuous-flow left ventricular assist devices - a systematic review
}

\author{
Elizabeth J. Maynes, Jonathan S. Gordon, Matthew P. Weber, Thomas J. O'Malley, Tyler M. Bauer, \\ Chelsey T. Wood, Rohinton J. Morris, Louis E. Samuels, John W. Entwistle, H. Todd Massey, \\ Vakhtang Tchantchaleishvili \\ Division of Cardiac Surgery, Thomas Jefferson University, Philadelphia, Pennsylvania, USA \\ Correspondence to: Vakhtang Tchantchaleishvili, MD. Assistant Professor of Surgery, Division of Cardiac Surgery, Thomas Jefferson University, 1025 \\ Walnut St, Suite 607, Philadelphia, PA 19107, USA. Email: Vakhtang.Tchantchaleishvili@jefferson.edu.
}

\begin{abstract}
Background: With increased use of continuous-flow left ventricular assist devices (CF-LVAD), development of malignant tumors in this population is not uncommon. We sought to evaluate malignancies in CF-LVAD patients and evaluate the outcomes of treatment strategies.

Methods: Overall, 18 articles consisting of 28 patients were identified who developed malignancies after CF-LVAD placement. Patient-level data were extracted for systematic review.

Results: Median patient age was 60 years [59-67] and 85.7\% (24/28) were male. CF-LVAD was placed as bridge-to-transplant (BTT) in $60.9 \%(14 / 23)$ of patients. The three most common malignancy types were GI in $35.7 \%(10 / 28)$ of patients, lung in $21.4 \%(6 / 28)$ and skin in $10.7 \%(3 / 28)$. Median time from CFLVAD implant to malignancy diagnosis was 6.9 [2.5-12.8] months. Metastatic disease occurred in $17.9 \%$ $(5 / 28)$ over a median time of 5.0 [1.0-82.0] months from the diagnosis. Surgical resection of the malignancy was performed in $57.1 \%(16 / 28)$ of patients. Our results showed that while there was a significantly higher probability of survival among patients who underwent surgery versus those who did not, when only stage I and II patients were included in the analysis, this difference was no longer statistically significant. Three patients were relisted for heart transplant after surgical treatment, and two received the transplant.

Conclusions: Surgical management of malignancies in patients on CF-LVADs may improve survival and transplant eligibility status, therefore, a CF-LVAD should not always preclude surgical treatment.
\end{abstract}

Keywords: Left ventricular assist devices; tumors; surgical resection; non-cardiac surgery

Submitted Apr 17, 2020. Accepted for publication Jul 24, 2020.

doi: 10.21037/acs-2020-cfmcs-10

View this article at: http://dx.doi.org/10.21037/acs-2020-cfmcs-10

\section{Introduction}

Mechanical circulatory devices have evolved over time from pulsatile devices associated with high rates of embolic events and device malfunction to continuous-flow left ventricular assist devices (CF-LVAD) characterized by lower adverse events and complications (1). While CF-LVADs were initially only used as a bridge-to-transplant (BTT), they are now being implanted in select patients for indefinite support as destination therapy (DT) with no intent of transplant or weaning from the device. Approximately half of patients who receive CF-LVADs have a DT indication (2) with the recent reported survival rate of $78 \%, 71 \%$, and $45 \%$ at 1 -, 2 - and 4-years respectively. With changes to indications and a reduced rate of complications that have come along with device advancements, patients with heart failure are living longer on mechanical circulatory support and therefore, the risk of developing concomitant non-cardiac diseases requiring surgery is more likely. Current research suggests that up to $27 \%$ of CF-LVAD patients undergo non-cardiac surgery, and this number is likely to rise as CF-LVADs 
continue to improve (3). This means management teams will be confronted with difficult decisions regarding both surgical and pharmacological interventions in patients with CFLVADs previously not considered. The development and surgical treatment of malignancies in CF-LVAD patients is one area of growing concern (4). Although some research has been published on non-cardiac surgery in patients on CF-LVADs, studies that focus on the development and surgical treatment of malignancies following CF-LVAD implantation are lacking (5-7).

The goal of this systematic review was to identify patterns in malignancy development following CF-LVAD placement and highlight specific approaches to treatment in this patient population.

\section{Methods}

\section{Literature search strategy}

A thorough systemic electronic search was performed in May 2020 using Cochrane Controlled Trials Register, Ovid Medline, Cumulative Index of Nursing and Allied Health Literature (CINAHL), and Scopus. To achieve the maximum sensitivity of the search strategy, the following combined terms were used: (heart assist device OR LVAD OR assisted circulation OR left ventricular assist device) AND (tumor OR tumour OR carcinoma OR adenocarcinoma OR adenoma OR dysplasia OR neoplasia OR malignancy) AND (resection OR mass OR chemotherapy OR radiotherapy OR radiation therapy OR radiofrequency ablation). The reference lists of all eligible studies were reviewed for further identification of potentially relevant studies and assessed using the inclusion and exclusion criteria.

\section{Selection criteria}

Eligible articles for the present systematic review included case reports/series that focused on malignancy development in patients following CF-LVAD implantation. Articles were excluded if they contained information on malignancy development before CF-LVAD implantation. Patients less than 18 years of age were also excluded. To ensure our results were reflective of current practice, only articles published over the last twelve years were included. Reports not published in the English language, abstracts, conference presentations, editorials, reviews and expert opinions were also excluded.

\section{Data extraction and critical appraisal}

Patient-level data were extracted from article texts, tables, and figures (EM, JG). Discrepancies between the reviewers were resolved by discussion and consensus. When data were not available, attempts were made to contact the corresponding authors to obtain the relevant data for the current study.

\section{Statistical analysis}

Baseline characteristics and demographics were reported using descriptive statistics, including medians and interquartile ranges (IQR) for continuous data and percentages for categorical data. Individual patient survival and outcome data from each case report and series were combined to produce Kaplan-Meier survival curves. Given the complexities underlying the treatment of malignancy, several curves were produced to indicate different subgroups to mitigate bias. These included the survival probability from the initiation of treatment for the malignancy, the survival probability for those who underwent surgery versus those who did not, and the survival probability for those with stage I and II disease who underwent surgical treatment versus those who underwent non-surgical treatment. As patients with a lower stage disease are more likely to be surgical candidates for curative resection, the stage III and IV patients were not included in the sub-analysis though one stage III patient, who was excluded from the analysis, did undergo surgical resection aimed at a cure. A cumulative hazard analysis was performed to assess development of metastatic disease. In an attempt to compare similarly staged patients, the one stage III patient was excluded and the sub-analysis was performed only on stage I and II patients. The remainder of the surgical group in stage III or IV underwent these procedures with a palliative mindset, those who underwent surgical resection versus those who had non-operative treatment such as chemotherapy and radiation. All analyses were performed through R software, version 3.5.1 (R Foundation for Statistical Computing, Vienna, Austria). P values $<0.05$ were considered statistically significant.

\section{Results}

\section{Study characteristics}

Overall, 1,873 articles were identified in the literature 


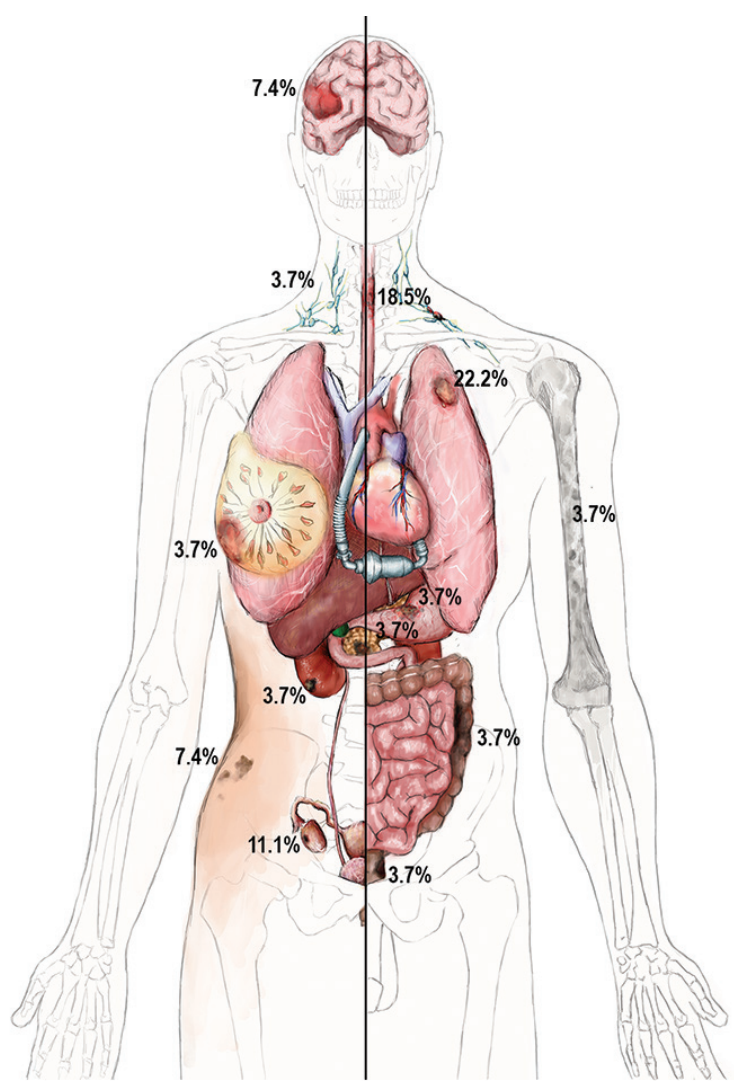

Figure 1 Anatomical illustration showing the organ distribution of malignancy development following CF-LVAD implantation. CFLVAD, continuous-flow left ventricular assist devices. Illustration by C.T.W.

search. Following application of the selection criteria, 13 publications remained for analysis. A manual search of references identified five more reports for a total of 18 case reports/series, consisting of 28 patients total included in the analysis. A PRISMA flow diagram depicting the overall search strategy is provided in Figure S1.

\section{Baseline demographics}

The median age of patients was 60.5 [59-67] years, $85.7 \%$ $(24 / 28)$ were male and $60.9 \%(14 / 23)$ of patients underwent CF-LVAD implantation as a BTT. The majority of patients received a HeartMate II LVAD $(70.6 \%, 12 / 17)$ and most patients $(64.3 \%, 18 / 28)$, had a history of ischemic heart disease. The demographic characteristics and indications are outlined in Table 1.

\begin{tabular}{ll}
\hline Table 1 Baseline demographics, CF-LVAD indication and etiology \\
\hline Demographics & Total $(\mathrm{n}=28)$ \\
\hline Male, $\mathrm{n}(\%)$ & $24 / 28(85.7)$ \\
\hline Age, median (IQR) & $60.0(58.0-65.5)$ \\
\hline CF-LVAD type, $\mathrm{n}(\%)$ & \\
\hline HeartMate II LVAD & $12 / 17(70.6)$ \\
HeartMate 3 LVAD & $1 / 17(5.9)$ \\
\hline HeartWare HVAD & $3 / 17(17.6)$ \\
\hline DuraHeart & $1 / 17(5.9)$ \\
Unspecified & $11(39.2)$ \\
\hline Indication, $\mathrm{n}(\%)$ & \\
\hline Bridge to transplant & $14(50)$ \\
\hline Destination therapy & $9(32.1)$ \\
\hline Unspecified & $5(17.9)$ \\
\hline Etiology, $\mathrm{n}$ (\%) & \\
\hline Ischemic heart disease & $18(64.3)$ \\
\hline Non-ischemic heart disease & $6(21.4)$ \\
\hline Unspecified & $414.3)$ \\
\hline CF-LVAD, continuous-flow left ventricular assist devices.
\end{tabular}

Time of LVAD support, malignancy characteristics and metastatic location

Details on malignancy types and distribution are shown in Table 2. Overall, 35.7\% (10/28) of patients had a gastrointestinal (GI) malignancy: $17.9 \%(5 / 28)$ had an esophageal malignancy, $3.6 \%(1 / 28)$ presented with colon, $3.6 \%(1 / 28)$ with rectal, $3.6 \%(1 / 28)$ with pancreatic and $3.6 \%(1 / 28)$ with a stomach malignancy. Overall, $21.4 \%$ $(6 / 28)$ of patients had a lung malignancy and $10.7 \%(3 / 28)$ were diagnosed with a skin malignancy: $7.1 \%(2 / 28)$ of patients presented with melanoma and one with basal cell carcinoma $(3.6 \%, 1 / 28)$. In addition, $7.1 \%(2 / 28)$ patients developed CNS tumors, $7.1 \%(2 / 28)$ had a renal malignancy, and there was one case (3.6\%) each of breast, ovary, prostate, lymphoma and multiple myeloma. The organ distribution of malignancy development is shown in the anatomical illustration (Figure 1). Table 3 provides additional information on tumor metastases location, histologic subtypes and primary site. 


\begin{tabular}{|c|c|}
\hline Tumor types and staging & Total $(n=28)$ \\
\hline \multicolumn{2}{|l|}{ Primary malignancy location } \\
\hline Gastrointestinal, n (\%) & $10(35.7)$ \\
\hline Esophagus, adenocarcinoma & $5(17.9)$ \\
\hline Rectum, adenocarcinoma & $1(3.6)$ \\
\hline Colon, adenocarcinoma & $2(7.1)$ \\
\hline Stomach, adenocarcinoma & $1(3.6)$ \\
\hline Pancreas, adenocarcinoma & $1(3.6)$ \\
\hline Lung, any, n (\%) & $6(21.4)$ \\
\hline Adenocarcinoma & $1(3.6)$ \\
\hline Renal cell carcinoma & $1(3.6)$ \\
\hline Squamous cell carcinoma & $1(3.6)$ \\
\hline Small cell carcinoma & $1(3.6)$ \\
\hline Unspecified & $2(7.1)$ \\
\hline Skin, any, n (\%) & $3(10.7$ \\
\hline Melanoma & $2(7.1)$ \\
\hline Basal cell carcinoma & $1(3.6)$ \\
\hline Kidney, any, n (\%) & $2(7.2)$ \\
\hline Renal cell carcinoma & $1(3.6)$ \\
\hline Transitional cell carcinoma & $1(3.6)$ \\
\hline Brain, any, n (\%) & $2(7.2)$ \\
\hline Glioblastoma multiforme & $1(3.6)$ \\
\hline Unspecified & $1(3.6)$ \\
\hline Breast, in situ ductal carcinoma, $\mathrm{n}(\%)$ & $1(3.6)$ \\
\hline Ovary, n (\%) & $1(3.6)$ \\
\hline Prostate, adenocarcinoma, n (\%) & $1(3.6)$ \\
\hline Lymphoma, n (\%) & $1(3.6)$ \\
\hline Multiple myeloma, $\mathrm{n}(\%)$ & $1(3.6)$ \\
\hline \multicolumn{2}{|l|}{ Cancer stage } \\
\hline \multicolumn{2}{|l|}{ Surgery*, n (\%) } \\
\hline Stages I-II & $7(53.8)$ \\
\hline Stages III-IV & $6(46.1)$ \\
\hline \multicolumn{2}{|l|}{ No surgery ${ }^{\star \star}, \mathrm{n}(\%)$} \\
\hline Stages I-II & $3(33.3)$ \\
\hline Stages III-IV & $6(66.7)$ \\
\hline
\end{tabular}

*, Out of 13 patients who received surgery with reported data on staging; ${ }^{* *}$, out of 9 patients who did not receive surgery with reported data on staging. CF-LVAD, continuous-flow left ventricular assist devices.

\begin{tabular}{lll}
\hline \multicolumn{2}{l}{ Table 3 Metastatic location, primary site and histologic subtype } \\
\hline Metastasis site & $\begin{array}{l}\text { Primary site/ } \\
\text { histologic subtype }\end{array}$ & $\begin{array}{l}\text { Total } \\
(\mathrm{n}=28)\end{array}$ \\
\hline All, n (\%) & - & $5(17.9)$ \\
\hline Lung, n (\%) & Renal cell carcinoma & $1(3.6)$ \\
Liver, n (\%) & Rectum, adenocarcinoma & $1(3.6)$ \\
\hline Omentum, n (\%) & Ovary & $1(3.6)$ \\
\hline Vertebra, n (\%) & Gl, adenocarcinoma & $1(3.6)$ \\
\hline Unspecified, n (\%) & Skin, melanoma & $1(3.6)$ \\
\hline
\end{tabular}

\begin{tabular}{|ll}
\hline \multicolumn{2}{|l}{ Table 4 Malignancy treatment types and frequencies } \\
\hline Treatment type & Total $(\mathrm{n}=28)$ \\
\hline Surgical resection, $\mathrm{n}(\%)$ & $16(57.1)$ \\
\hline Radiation, $\mathrm{n}(\%)$ & $5(17.9)$ \\
\hline Radiofrequency ablation & $1(3.6)$ \\
\hline Stereotactic body radiation therapy & $1(3.6)$ \\
\hline Chemotherapy, $\mathrm{n}(\%)$ & $3(10.7)$ \\
\hline Chemoradiation, $\mathrm{n}(\%)$ & $2(7.1)$ \\
\hline Surgery and chemotherapy, $\mathrm{n}(\%)$ & $1(3.6)$ \\
\hline Surgery, chemotherapy and radiation, $\mathrm{n}(\%)$ & $1(3.6)$ \\
\hline Placed on waiting list, $\mathrm{n}(\%)$ & $3(10.7)$ \\
\hline Eventually transplanted & $2(7.1)$ \\
\hline Palliative care, $\mathrm{n}$ (\%) & $2(7.1)$ \\
\hline
\end{tabular}

\section{Malignancy types, frequency and duration of CF-LVAD support}

Of all patients, $57.1 \%(16 / 28)$ received surgical treatment including $50.0 \%(14 / 28)$ who received surgical treatment only, $3.6 \%(1 / 28)$ received chemotherapy in addition to surgical treatment, and $3.6 \%(1 / 28)$ received a combination of chemotherapy, radiation and surgical treatment. The remaining patients did not receive surgery for treatment and instead $35.7 \%(10 / 28)$ underwent either radiation therapy, chemotherapy, chemoradiation or radiofrequency ablation, and $7.1 \%(2 / 28)$ received palliative care only (Table 4, Figure 2). The time in months that patients were on CF-LVAD support before and after the malignancy diagnosis is outlined in Table 5 . 


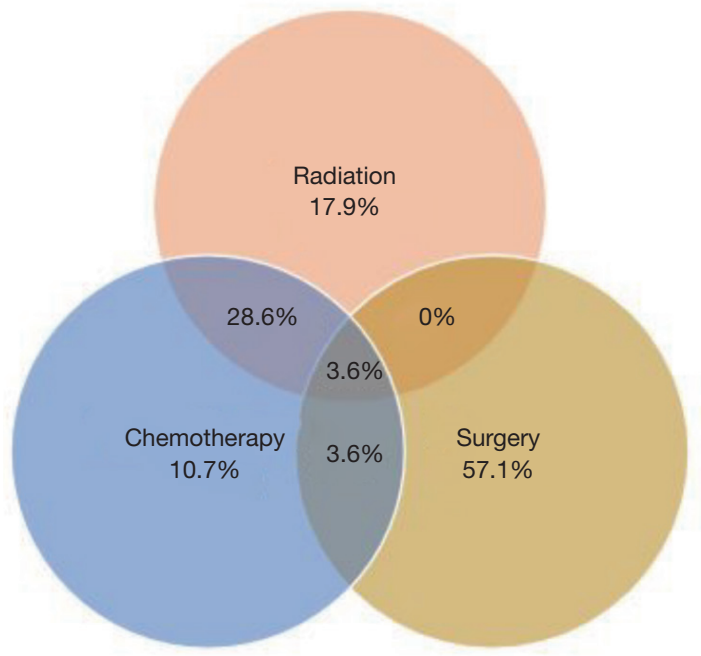

Figure 2 Venn diagram indicating the frequency of each treatment approach.

\begin{tabular}{|c|c|}
\hline Variable & Total $(n=24)$ \\
\hline $\begin{array}{l}\text { Total duration of CF-LVAD } \\
\text { support in months, median (IQR) }\end{array}$ & $33.9(17.8,45.3)$ \\
\hline $\begin{array}{l}\text { CF-LVAD support before malignancy } \\
\text { diagnosis in months, median (IQR) }\end{array}$ & $6.9(2.5,12.8)$ \\
\hline $\begin{array}{l}\text { Malignancy treatment to metastasis } \\
\text { in months, median (IQR) }\end{array}$ & $12(5.9,24.0)$ \\
\hline $\begin{array}{l}\text { Malignancy treatment to recent } \\
\text { follow-up in months, median (IQR) }\end{array}$ & $13.8(5.9,27.3)$ \\
\hline
\end{tabular}

\section{Survival}

Data were analyzed to assess the probability of patient survival at various stages of the clinical course following CF-LVAD implantation. A Kaplan-Meier graph indicating the probability of survival from initiation of CF-LVAD support is shown in Figure 3. Data were also analyzed to assess the probability of patient survival from the initiation of malignancy treatment to the most recent follow-up (Figure 4) and to evaluate probability of survival stratified by patients treated surgically versus those who received other forms of treatment (Figure 5). There appeared to be

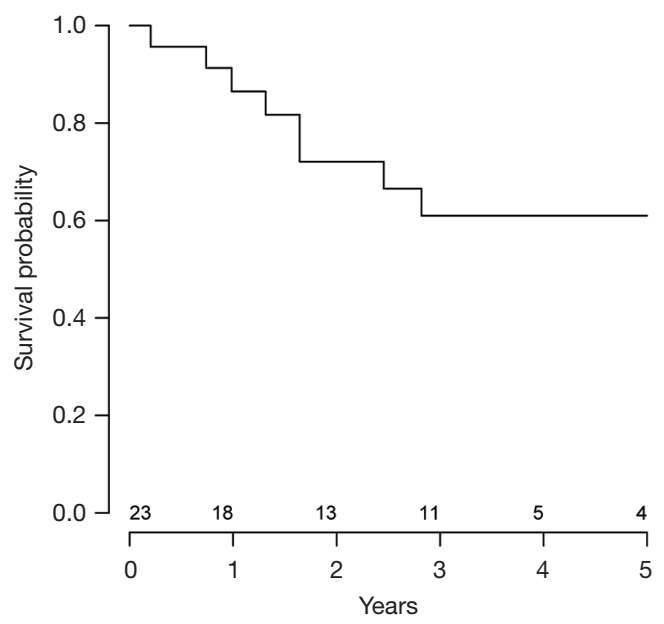

Figure 3 Kaplan-Meier graph indicating survival probability from initiation of CF-LVAD support. CF-LVAD, continuous-flow left ventricular assist devices.

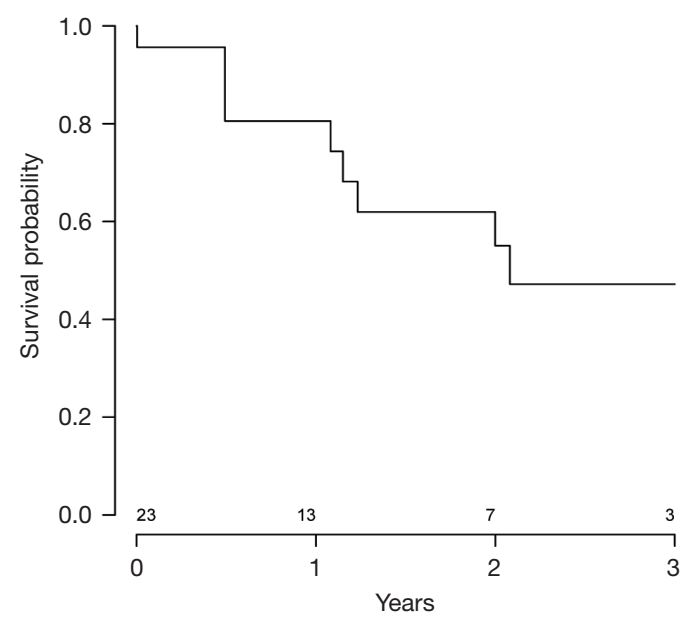

Figure 4 Kaplan-Meier graph indicating survival probability from the initiation of treatment for the malignancy.

a higher probability of survival over the 3-year period for those who had surgery versus those who did not $(\mathrm{P}=0.05)$; however, when patients with stage I and II who underwent surgery versus those with stage I and II who did not receive surgery (Figure 5) were compared, this difference was no longer statistically significant. $(\mathrm{P}=0.07)$. Out of all the patients, $17.9 \%(5 / 28)$ had evidence of metastatic disease. A cumulative hazard plot representing the pattern of metastatic disease development over time is shown in Figure 6. 

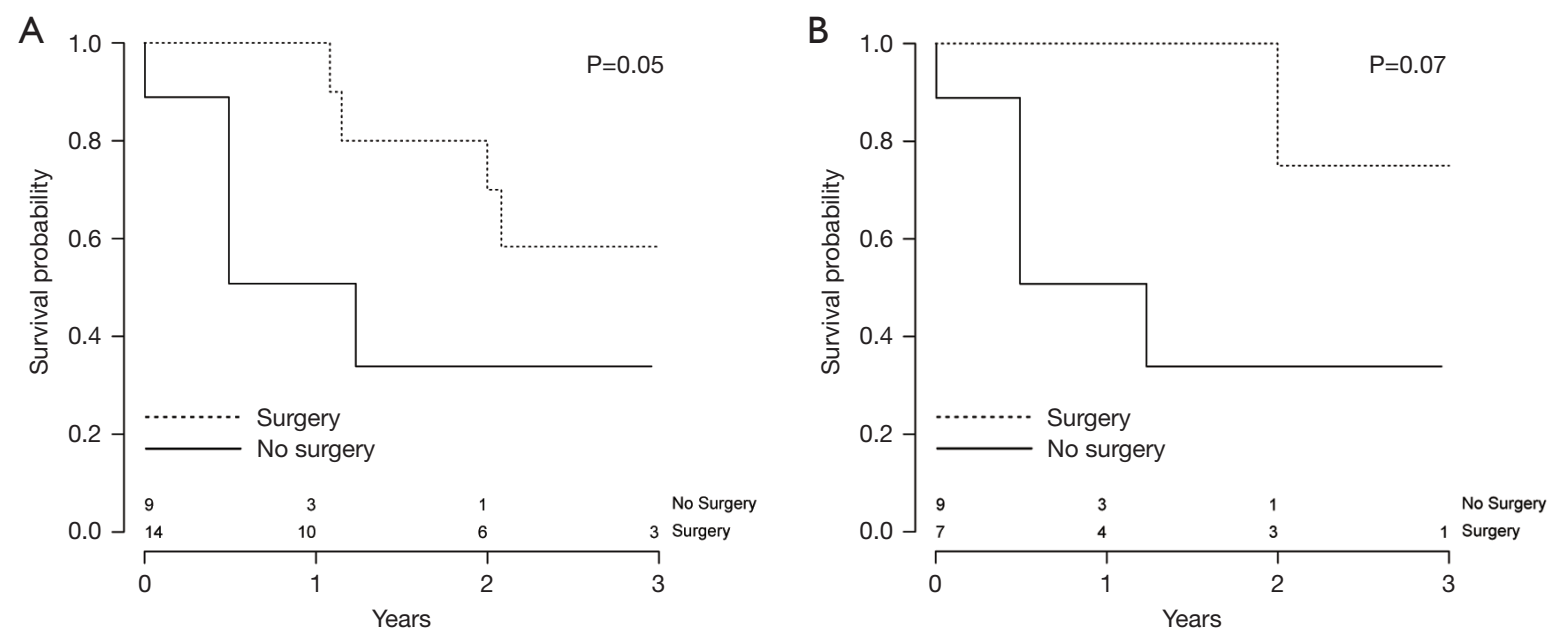

Figure 5 Stratified Kaplan-Meier curve indicating survival probability for those who underwent surgery versus those who received nonsurgical treatment. (A) All patients (B) patients with stage I and II.

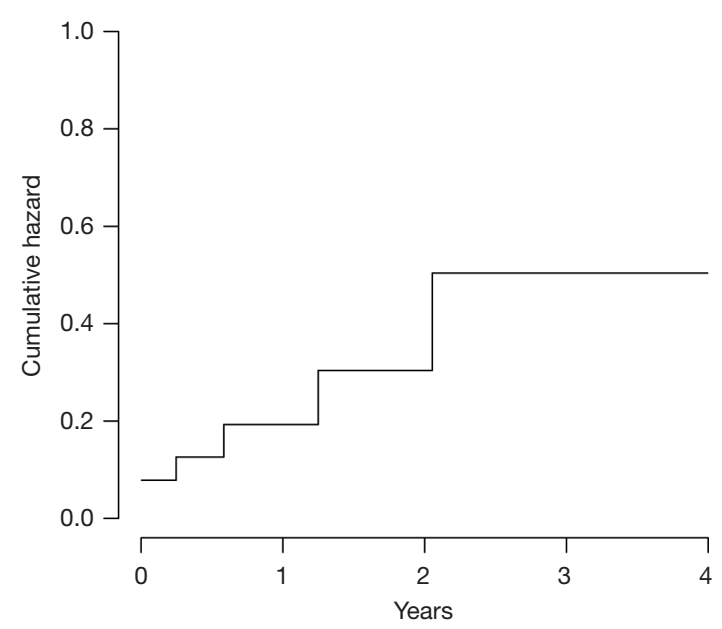

Figure 6 Cumulative hazard plot showing the risk of developing metastatic disease following the initial treatment.

\section{Discussion}

In our cohort of 28 patients with malignancy development following CF-LVAD placement, we found that a large majority of patients developed gastrointestinal malignancies. We also found that there was a significantly higher probability of survival among patients who underwent surgery versus those who did not. However, when only stage I and II patients who received surgery were compared to stage I and II patients who did not receive surgery, the differences between these two groups were no longer significant. Given the limitation of a lower patient number with respect to this analysis, it is possible this change is a reflection of the lower patient numbers in each subgroup.

Malignancy development and treatment following device implantation brings up important issues regarding patient eligibility for a heart transplant. Most pre-transplant assessment programs will avoid heart transplantation in patients who have had or present with a primary or secondary malignancy (8). However, as mentioned by Mehra et al., since pre-existing malignancies are diverse and many are treatable, patients should be stratified according to their risk of malignancy recurrence (9). ISHLT guidelines recommend that cardiac transplantation be considered when malignancy recurrence is low based on type, response to therapy and negative metastatic work-up. They also suggest that no arbitrary time period for observation should be used in the heart transplant decision making process for patients with a history of malignancy (Level of Evidence: C) (9). Table 4 gives the number of patients who were put on the waiting list for heart transplantation and the percentage of those who were eventually transplanted following treatment of their malignancy. The variation in baseline characteristics of patients with different malignancies and type-specific responses to heart transplantation management can make the patient's clinical course and future outcomes following transplantation largely unpredictable (8). Factors such as recurrence rates, metastatic potential and the interaction with immunosuppression regimens should be taken into consideration in evaluating and proceeding with 
transplantation.

However, even if malignancies are treated and the patient eventually undergoes a heart transplant, there is a potential increased risk of tumor development following heart transplantation. Having a previous tumor can increase the risk for recurrence by 1.8 times in heart transplant recipients and is associated with a significantly lower overall survival 10 years postoperatively compared to those with no previous malignancy (10).

While no cause and effect relationship has been identified between CF-LVAD implantation and malignancy development, there is some evidence for transitory immunologic changes after CF-LVAD implantation including an initial pronounced apoptosis-specific immune alteration by increased annexin $\mathrm{V}$ binding to $\mathrm{CD} 3 \mathrm{~T}$ cells and death-inducing receptors soluble CD95/tumor necrosis factor-R1 (11). Additionally, correlations between device implantation, end-stage cardiomyopathies and implications for malignancy development have been identified in the past (12-15). Further, large-scale studies investigating the immunologic alterations after CF-LVAD implantation in end-stage heart failure patients are warranted.

GI and lung malignancies were the most common malignancy types in patients following CF-LVAD implantation, a finding that does not deviate substantially from current trends in malignancy types affecting older patients (16). For those patients on a CF-LVAD who underwent surgery for treatment of a GI malignancy, abdominal incisions for visceral malignancies and associated technical difficulties due to the CF-LVAD pump or the driveline located transversely in the preperitoneal space were discussed (17-19). As most drivelines are situated in the horizontal line with the umbilicus, any procedure that involves the Maylard incision (many pelvic and abdominal surgeries) would be particularly affected by the driveline exit site. In one patient with a pancreatic malignancy, the incision for the pancreatic tumor was altered from the standard midline supraumbilical incision in order to avoid the driveline and the CF-LVAD pump location within the abdominal wall; the location of the driveline and identification of vital arteries including the superior mesenteric, celiac, hepatic, and gastroduodenal artery were also carefully assessed (19).

As shown in Table 4, 16/28 (57.1\%) of patients underwent surgery regardless of the risks associated with CF-LVADs. Hemodynamics, risk of bleeding, age and history of previous cardiac surgery were factors all taken into consideration in determining whether a patient qualified for surgery $(20,21)$. Studies show that elective surgical procedures in patients with CF-LVADs may be performed with an acceptable risk if the operation is carefully managed $(22,23)$. In order to determine the appropriate treatment plan for these complex patients, collaborative, multidisciplinary meetings that include surgeons, cardiologists, cardiac anesthesiologists, and oncologists should be employed (17).

Our results regarding post-surgical survival and transplant outcomes are comparable to past studies on non-cardiac surgical procedures in CF-LVAD patients. In 2009, Stehlik et al. analyzed 184 patients on CF-LVADs and found that 37 patients (24\%) subsequently required 59 non-cardiac surgeries; a relatively low 30-day mortality rate of $12 \%$ was reported with none of the deaths directly from the non-cardiac surgery. Of this group, $72 \%$ of those being BTT were successfully transplanted, which was slightly higher than those who did not have surgery $(6,22)$. In 2009, Brown et al. also compared outcomes of patients on CF-LVADs who required non-cardiac surgery and found that although complications such as risk of infection and bleeding were common, there appeared to be no increased mortality risk when compared to those with CF-LVADs who did not require surgery (24). However, a more recent review indicated that general surgical complications associated with use of long-term mechanical circulatory support contributed significantly to morbidity and mortality in these patients (25). While the presence of a CF-LVAD was cause for concern in non-cardiac surgery in the past, subsequent advances in CF-LVAD technology led to changes that have been beneficial in regards to non-cardiac surgery. This includes transition to intrapericardial pump placement from the pre-peritoneal abdominal location, and overall increase in knowledge base in perioperative management of CF-LVAD patients with adoption of standardized treatment protocols. However, alterations in coagulation and platelet function secondary to large molecular weight $\mathrm{vWF}$ destruction still remain problematic $(26,27)$.

As mentioned in the reports, when a patient's disease was too advanced for surgery or if the patient presented with metastasis, alternative options such as chemotherapy, radiotherapy or palliative care were chosen $(20,21,28)$. The effects of radiation on CF-LVADs were discussed by Emerson et al. and Ostertag et al. $(29,30)$ who suggested that the rotor and rotor magnets within the CF-LVAD were of particular concern in patients receiving radiation. Recommendations for CF-LVAD protection during 
radiotherapy include protecting the CF-LVAD battery source by placing it outside the RT field. It has been shown that the CF-LVAD may remain unaffected by the irradiation if doses are kept $\leq 70$ Gy (29). In vitro studies to assess the potential electronic instability of the HeartWare HVAD device have been conducted by Gossman et al. who evaluated the direct effects of irradiation from megavoltage $\mathrm{x}$-rays provided by a radiation oncology particle accelerator (31). Overall, they found that an HVAD pump was not affected by radiation exposure at clinically relevant doses and that current treatment planning software underestimated dosage delivery. They concluded that their study provided a maximal dosage applied at a frequent number of iterations, but it could not predict the true longterm effects of prolonged radiation treatment (31). Further studies that assess radiation effects on CF-LVAD function including those that compare intracorporeal (such as HeartMate 3) vs extracorporeal (HeartMate 2) controllers are warranted.

Patients in whom malignancies are detected following CF-LVAD implantation belong to a unique category that falls outside the normal DT or BTT groups. Therefore, a more tailored patient specific approach is needed for this unique group of patients.

\section{Limitations}

This systematic review has several key limitations and must be interpreted with care. Since this study was based on case reports with a limited number of patients included, there was a lack of high-quality data available on patient management, which led to a heterogeneous reporting of outcomes. For the Kaplan-Meier sub-analysis that included only those with stage I and II disease, the number of patients was low and hence this likely affected the power of the study. In general, patients with lower stage disease, as is the case for stage I and II disease, are more likely to be surgical candidates for curative resection. Some patients with stage III or IV disease did undergo surgical treatment with a majority of these cases being palliative in nature. However, one of the patients in this study with stage III disease underwent an operation aimed at a cure. In an attempt to compare similarly staged patients, this one stage III patient was excluded and the sub-analysis was performed only on stage I and II patients. Furthermore, data were not available to assess differences in outcomes based on the etiology of the primary tumor due to these limited numbers. Therefore, further investigations that include larger patient cohorts are warranted. We were also unable to calculate the incidence of malignancies in this patient population since all patients included had a diagnosis of a malignancy. Despite these limitations, this research systematically assessed the development of malignancy following CF-LVAD implantation, various treatment options, and survival outcomes among this patient population. It is possible that frequent CT scans, upper and lower endoscopies, and other surveillance testing that these patients undergo helped to identify some of these malignancies at an early stage, thereby prompting earlier treatment and perhaps better survival. There may be some heterogeneity between the populations undergoing surgical interventions and non-surgical interventions, potentially due to the different disease severities of the patients which necessitated these interventions. Future studies into this subject may result in more granular data to allow for subanalyses between the surgical and nonsurgical subgroups in the management of CF-LVAD patients with malignancies who require surgical treatment. Unfortunately, with limited studies currently available for analysis and relatively small cohorts of patients included, this was not feasible at the current time.

In conclusion, our results showed that there was a significantly higher probability of survival among patients who underwent surgery versus those who did not and when only stage I and II patients were included in the analysis, the differences were no longer significant. Therefore, placement of a CF-LVAD should not preclude patients from receiving surgical treatment for malignancy as it may help patients achieve heart transplant eligibility status. Future studies investigating pre- and post-implantation malignancy screening and possible immunologic modulation associated with CF-LVAD are warranted.

\section{Acknowledgments}

Funding: None.

\section{Footnote}

Conflicts of Interest: The authors have no conflicts of interest to declare.

Open Access Statement: This is an Open Access article distributed in accordance with the Creative Commons Attribution-NonCommercial-NoDerivs 4.0 International License (CC BY-NC-ND 4.0), which permits the non- 
commercial replication and distribution of the article with the strict proviso that no changes or edits are made and the original work is properly cited (including links to both the formal publication through the relevant DOI and the license). See: https://creativecommons.org/licenses/by-nc-nd/4.0/.

\section{References}

1. Slaughter MS, Rogers JG, Milano CA, et al. Advanced Heart Failure Treated with Continuous-Flow Left Ventricular Assist Device. N Engl J Med 2009;361:2241-51.

2. Fukunaga N, Rao V. Left ventricular assist device as destination therapy for end stage heart failure: the right time for the right patients. Curr Opin Cardiol 2018:33:196-201.

3. Barbara DW, Wetzel DR, Pulido JN, et al. The Perioperative Management of Patients With Left Ventricular Assist Devices Undergoing Noncardiac Surgery. Mayo Clin Proc 2013;88:674-82.

4. Loyaga-Rendon RY, Inampudi C, Tallaj JA, et al. Cancer in end-stage heart failure patients supported by left ventricular assist devices. ASAIO J 2014;60:609-12.

5. Nelson JA, Mauermann WJ, Barbara DW. Left Ventricular Assist Devices and Noncardiac Surgery. Adv Anesth 2018;36:99-123.

6. Stehlik J, Nelson DM, Kfoury AG, et al. Outcome of noncardiac surgery in patients with ventricular assist devices. Am J Cardiol 2009;103:709-12.

7. Taghavi S, Beyer C, Vora H, et al. Noncardiac Surgery in Patients on Mechanical Circulatory Support. ASAIO J 2014;60:670-4.

8. Chapman JR, Webster AC, Wong G. Cancer in the Transplant Recipient. Cold Spring Harb Perspect Med 2013;3:a015677.

9. Mehra MR, Canter CE, Hannan MM, et al. The 2016 International Society for Heart Lung Transplantation listing criteria for heart transplantation: A 10-year update. J Heart Lung Transplant 2016;35:1-23.

10. Delgado JF, Alonso-Pulpón L, Mirabet S, et al. Cancer Incidence in Heart Transplant Recipients With Previous Neoplasia History. Am J Transplant 2016;16:1569-78.

11. Ankersmit HJ, Wieselthaler G, Moser B, et al. Transitory immunologic response after implantation of the DeBakey VAD continuous-axial-flow pump. J Thorac Cardiovasc Surg 2002;123:557-61.

12. McComb JM. Implanted defibrillators and cancer: the power of registries. Europace 2015;17:1741-2.

13. Keel SB, Jaffe KA, Nielsen GP, et al. Orthopaedic
Implant-Related Sarcoma: A Study of Twelve Cases. Mod Pathol 2001;14:969-77.

14. Adams JE, Jaffe KA, Lemons JE, et al. Prosthetic implant associated sarcomas: A case report emphasizing surface evaluation and spectroscopic trace metal analysis. Ann Diagn Pathol 2003;7: 35-46.

15. Khamooshian A, Klinkenberg TJ, Maass AH, et al. Management of device-related malignant sarcoma. HeartRhythm Case Rep 2017;3:373-6.

16. Pilleron S, Sarfati D, Janssen-Heijnen M, et al. Global cancer incidence in older adults, 2012 and 2035: A population-based study. Int J Cancer 2019;144:49-58.

17. Nakamura Y, Toda K, Nakamura T, et al. Curative surgery for gastric cancer in a patient with an implantable left ventricular assist device J Artif Organs 2017;20:170-3.

18. Zarbaliyev E, Balkanay M, Sarsenov D. Embracing the Future of Surgery: Gastric Cancer Resection Within One Month of Left Ventricular Assist Device Implantation. Cureus 2018;10:e2868.

19. Mejia JC, Dong M, Ojogho O. Pancreaticoduodenectomy in a patient with previous left ventricular assist device: a case report with specific emphasis on peri-operative logistics. J Surg Case Rep 2017;2017:rjx053.

20. Aday U, Gündeş E, Ali Çetin D, et al. Unexpected diagnosis in a patient with a left ventricular assist device: rectal cancer. Arch Med Sci Atheroscler Dis 2017;2:e31-3.

21. Samuels L, Casanova-Ghosh E, Domsky S, et al. Survival of the Unfittest: A case of End-Stage Heart Failure and advanced esophageal cancer managed successfully with an implantable Left Ventricular Assist Device (LVAD) and aggressive chemoradiation therapy. New Horizons Clin Case Reports 2017;2:20-1.

22. Schmid C, Wilhelm M, Dietl KH, et al. Noncardiac surgery in patients with left ventricular assist devices. Surgery 2001;129:440-4.

23. Stone M, Hinchey J, Sattler C, et al. Trends in the Management of Patients With Left Ventricular Assist Devices Presenting for Noncardiac Surgery. Semin Cardiothorac Vasc Anesth 2016;20:197-204.

24. Brown JB, Hallinan WM, Massey HT, et al. Does the need for noncardiac surgery during ventricular assist device therapy impact clinical outcome? Surgery 2009;146:62733; discussion 633-4.

25. Tchantchaleishvili V, Umakanthan R, Karp S, et al. General surgical complications associated with the use of long-term mechanical circulatory support devices: are we "under-reporting" problems? Expert Rev Med Devices 2013;10:379-87. 
26. Jilma-Stohlawetz P, Quehenberger P, Schima H, et al. Acquired von Willebrand factor deficiency caused by LVAD is ADAMTS-13 and platelet dependent Thromb Res 2016;137:196-201.

27. Bartoli CR, Restle DJ, Zhang DM, et al. Pathologic von Willebrand factor degradation with a left ventricular assist device occurs via two distinct mechanisms: Mechanical demolition and enzymatic cleavage. J Thorac Cardiovasc Surg 2015;149:281-9.

28. Khan M, Wasim A, Mirrakhimov AE, et al. Case Report of a Patient with Left Ventricular Assistance Device Undergoing Chemotherapy for a New Diagnosis of Lung
Cancer. Case Rep Oncol Med 2015;2015:163727.

29. Emerson LY, Deek MP, Almendral J, et al. Radiation therapy in patients with left ventricular assist device: A case report and literature review. Pract Radiat Oncol 2016;6:e145-7.

30. Ostertag-Hill CA, Mudd J, Werle DP, et al. Safe delivery of lung stereotactic body radiation therapy in a patient with a left ventricular assist device and implantable cardioverter defibrillator. Clin Case Rep 2018;6:1704-7.

31. Gossman MS, Graham JD, Tamez D, et al. Evaluation of a Ventricular Assist Device. ASAIO J 2012;58:212-6.
Cite this article as: Maynes EJ, Gordon JS, Weber MP, O'Malley TJ, Bauer TM, Wood CT, Morris RJ, Samuels LE, Entwistle JW, Massey HT, Tchantchaleishvili V. Development of malignancies and their outcomes in patients supported on continuous-flow left ventricular assist devices-a systematic review. Ann Cardiothorac Surg 2021;10(3):301-310. doi: 10.21037/acs-2020-cfmcs-10 


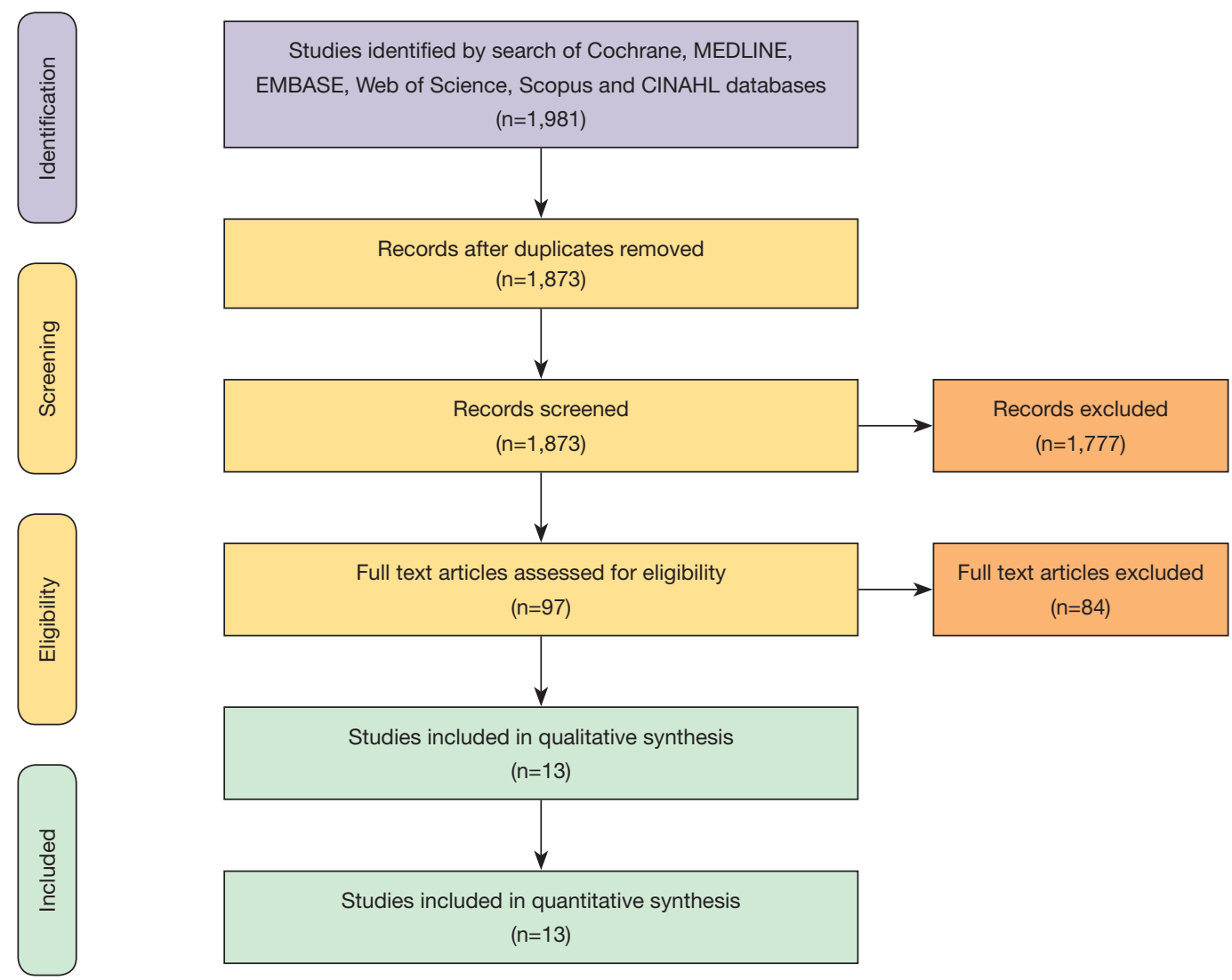

Figure S1 PRISMA schematic of the search strategy. PRISMA, Preferred Reporting Items for Systematic Reviews and Meta-Analyses. 